\title{
HYBRID SIMULATIONS OF PLASMASPHERIC REFILLING INCLUDING CONVECTION AND INJECTION
}

\author{
R. H. Miller,* C. E. Rasmussen,* T. I. Gombosi* and \\ D. Winske** \\ * Space Physics Research Laboratory, Department of Atmospheric, Oceanic \\ and Space Sciences, The University of Michigan, Ann Arbor, MI 48109-2143, \\ U.S.A. \\ ** Applied Theoretical Physics Division, Los Alamos National Laboratory, \\ Los Alamos, NM 87545, U.S.A.
}

\begin{abstract}
The interactions between upflowing thermal ions from conjugate ionospheres are modeled using a 1dimensional hybrid particle code. The simulation model allows for multiple species, convection of plasmaspheric flux tubes, and Coulomb self collisions which conserve momentum and energy. Plasmaspheric refilling is studied, in which convection of the plasmaspheric flux tube and particle injection from an external source is included. The interaction of ionospheric thermal plasma and the injected plasma seems to play an important role in the evolution of the total particle distribution on the early time scales $(t<1$ hour).
\end{abstract}

\section{INTRODUCTION}

The refilling of depleted plasmaspheric flux tubes is a dynamical process that has received much attention over the past several decades. The plasmapause boundary was first observed by Gringauz (1963) /1/ and Carpenter (1963) /2/. During magnetically quiet conditions $/ 3 /$, the plasmasphere is located Earthward of $\mathrm{L} \approx 5-6$ with a relatively high plasma density $\left(10^{2}\right.$ to $\left.10^{3} \mathrm{~cm}^{-3}\right) / 4 /$. The density decreases by two orders in magnitude at the plasmapause boundary and was called "Carpenters knee" to symbolically illustrate this fact $/ 3 /$. Plasmaspheric flux tubes are dominated by Earth's co-rotational electric field and are continually supplied by fluxes from the ionosphere until a diffusive equilibrium is obtained. The plasmapause density gradient is thought to be formed by peeling off the relatively dense plasmasphere due to variable magnetospheric convection (geomagnetic conditions). Flux tube trajectories just outside of the plasmapause are dominated by the magnetospheric convection electric field and can intersect the magnetopause and become open, thereby venting their plasma into interplanetary space. The "open" field lines are convected across the polar cap, reconnect in the tail of the magnetosphere and are convected sunward. The resulting supersonic flows from the ionosphere during the "venting" stage constitute the polar wind /5/. Refilling begins after reconnection in the magnetosphere while the flux tube is convected sunward.

In this paper, we present a hybrid model of plasmaspheric refilling, including the effects of Coulomb 
collisions, which model in a "heuristic" manner the dynamic scenario mention above concerning plasmaspheric refilling. An $\mathrm{L}=4$ flux tube with the plasma in diffusive equilibrium is convected to an $\mathrm{L}$ shell of 10 , and the connection to the conjugate ionosphere is broken. This provides an outer boundary (equator in the simulation) through which all particles that flow across are absorbed and, by maintaining the flux from the ionosphere, a "polar" wind solution along the separated flux tube is produced. A flux through the outer boundary of the disconnected flux tube is assumed, simulating the access of the field line to solar wind particles. The flux tube is reconnected after 4 hours and convected to $L=4$.

We begin in section 2 of this paper with a brief overview of the simulation model, while in section 3 we present our results and summarize our principal findings.

\section{SIMULATION METHOD}

The hybrid simulation code is electrostatic and treats the electrons as a massless, isothermal, charge neutralizing fluid and the ions as discrete particles. Since the electrons are massless, quasi-neutrality is maintained and the polarization electric field is obtained from the zero mass limit of the electron momentum equation. The guiding centers of the ions are followed in time and space, and the interaction between the ions and the electric field is calculated self-consistently. The discrete guiding centers of the ions are acted upon by the gravitational, magnetic mirror, and ambipolar electric forces. The simulation is one-dimensional in space, but the particle velocities are two-dimensional. The simulation domain is parallel to the magnetic field, which is assumed to be dipolar, so that an $\mathrm{L}$ shell is specified in each case.

Our notation and definitions are consistent, for the most part, with those of Miller et al. (1992) /6/. The base of the flux tube, where $\mathrm{H}^{+}$ions are injected, is taken to be $1.3 \mathrm{RE}_{\mathrm{E}}$ or $1900 \mathrm{~km}$. The density and temperature for the drifting distribution function are $n_{0}=400 \mathrm{~cm}^{-3}$ and $T_{0}=3000^{\circ} \mathrm{K}$, respectively, where the subscript " 0 " refers to values at a reference altitude of $1900 \mathrm{~km}$. The effects of small angle Coulomb collisions are included in the model using a method developed by Takizuka and Abe (1977) $7 /$ (See reference $/ 6 /$ ).

\section{RESULTS AND SUMMARY}

In this section, results from the two simulations are presented. The simulations start with an $\mathrm{L}=4$ plasmaspheric flux tube near diffusive equilibrium, and then convection and injection of ions is considered. All of the physical and simulation parameters (given in the proceeding section) are kept constant. Only the boundary condition and $\mathrm{L}$ shell number are varied.

The convection of the plasmaspheric flux tube has two dominant effects. The first is a change in the magnetic field strength (increase/decrease) which introduces a change in the cross-sectional area (decrease/increase) and, through the constancy of $\mu$, a change in the perpendicular temperature (increase/decrease). The second effect is the introduction of an inertial force parallel to the magnetic field due to the changing orientation of the magnetic field unit vector $/ 6 \%$. For convection to larger or smaller $L$ shell values, the inertial force introduces an acceleration towards the equator like a "sling shot": this force can be thought of as a centrifugal force.

We model the flux that would be obtained from the solar wind or the low latitude boundary layer (LLBL) for 30 minutes and then assume a zero flux boundary condition for the solar wind. The simulation by no means models the complicated dynamics encountered in the cleft/cusp regions but attempts to examine the nature of flows and how they influence some of the dynamics of plasmaspheric refilling. Once the flux tube is broken, the flux from the LLBL is assumed to be half of a non-drifting Maxwellian with a density and temperature of 30 particle $\mathrm{cm}^{-3}$ and $100 \mathrm{eV}$ for the ions $/ 8 /$, producing a flux of $1.17 \times 10^{8}$ particle $\mathrm{cm}^{-2} \mathrm{~s}^{-1}$. The flux from the equator is 1.48 times larger than the flux from the ionosphere. After 30 minutes the flux from the equator is zero and the plasma is absorbed at the equator for 3.5 hours. The flux tube is then reconnected and convected to an $L=4$. 
The particle distributions as a function of normalized parallel (Figures 1a-1c) and perpendicular (Figures 1d-1f) velocity and normalized distance along the flux tube are shown in Figure 1 for three different times: 4.00, 4.19, 4.34 hours after the flux tube has reached an $L$ shell of 10. Figures 1a and 1d shows the northern and southem particle distribution 4 hours after the flux tube has reached an $L$ shell of 10. The flux from the outer boundary produces an electric field directed from that boundary toward the ionosphere. This electric field inhibits the escape of particles from the flux tube, producing a higher flux tube content than in the absence of particle injection. Figure $1 \mathrm{~b}$ shows a beam velocity in excess of the thermal velocity with a Mach flow of 15 . By 4.34 hours the peak velocity has decreased in magnitude and is found to be $\pm 11 \mathrm{v}_{\text {th }}$ at the ionosphere in Figure 1c. The mechanism responsible for the increase in acceleration of the beam is the inertial force from the changing orientation of the magnetic field unit vector under convection. The low energy population during convection from an $L$ shell of 10 to 4 has moved from the equator to the ionosphere with velocities centered about $6 v_{\text {th }}$. shown in Figure 1c. Perpendicular heating of the plasma from convection (due to the constancy of $\mu$, the first adiabatic invariant) is shown in Figure 1f. The effective perpendicular temperature of the plasma near the ionosphere is $25 \mathrm{~T}_{0}$, considerably larger than the ionospheric temperature. Convection can play an important role in heating or cooling the plasma through conservation of the first adiabatic invariant.

Acknowledgments: This work was supported at the University of Michigan by the National Aeronautics and Space Administration (NASA) grants NAGW-2162 and NAGW-1619 and by the National Science Foundation under contract ATM-9114409. Research at Los Alamos was done under the auspices of the U. S. Department of Energy and was supported in part by the National Aeronautics and Space Administration Space Plasma Theory Program.

\section{REFERENCES}

1. K. I. Gringauz, The structure of the ionized gas envelope of the Earth from direct measurements in the U.S.S.R. of local charge particle concentrations, Planet. Space Sci., 11, 281, 1963.

2. D. L. Carpenter, Whistler evidence of a "knee" in the magnetospheric ionization density profile, J. Geophys. Res., 68, 1675, 1963.

3. C. R. Chappell, Recent satellite measurements of the morphology and dynamics of the plasmasphere, Rev. Geophys., 10, 951, 1972.

4. D. L. Carpenter, and C. G. Parks, On what ionospheric works should know about the plasmapause-plasmasphere, Rev. Geophys., 11, 133, 1973.

5. P. M. Banks, and T. E. Holzer, High-latitude plasma transport: the polar wind, J. Geophys. Res., 74, 6317, 1969.

6. G. R. Wilson, J. L. Horwitz, and J. Lin, A semi-kinetic model for early stage plasmaspheric refilling 1. Effects of Coulomb collisions, J. Geophys. Res., 97, 1109, 1992.

7. R. H. Miller, C. E. Rasmussen, T. I. Gombosi, and D. Winske, A kinetic simulation of plasma flows in the inner magnetosphere, J. Geophys. Res., submitted, 1992.

8. T. Takizuka, and A. Hirotada, A binary collision model for plasma simulation with a particle code, J. Comp. Phy., 25, 205, 1977.

9. J. C. Foster, J. M. Holt, R. G. Musgrove, and D. S. Evans, Ionospheric conyection associated with discrete levels of particle precipitation, Geophys. Res. Lett., 13, 656, 1986.

10. P. Song,, R. C. Elphic, C. T. Russell, J. T. Gosling, and C. A. Cattell, Structure and properties of the subsolar magnetopause for northward IMF: ISEE observations, J. Geophys. Res., 95, 6375, 1990. 


\section{Particle Distribution}
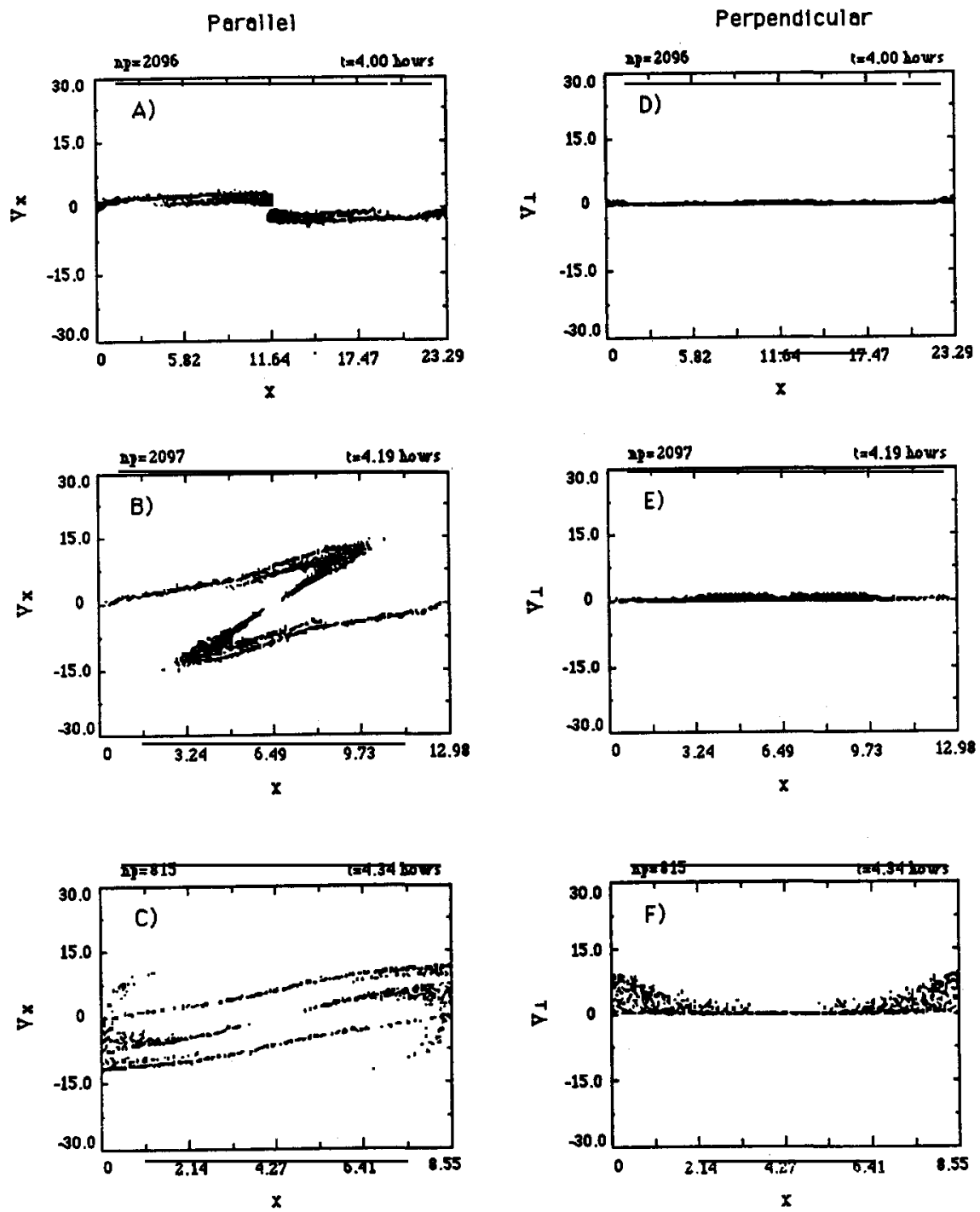

Figure 1: Figures 1a-1c and 1d-1f show the particle distribution as a function of parallel and perpendicular velocity and distance along the flux tube, respectively, at 3 sequential times in the simulation for a particle flux assumed at the equator. Figure 1a-1c and 1d-1f show the distributions at $4.00,4.19$, and 4.34 hours after the flux tube has reached an $\mathrm{L}$ shell of 10 . 